\title{
Digestibilidade e metabolismo de dietas de suínos contendo zearalenona com adição de organoaluminossilicato
}

\author{
Luciano Hauschild(1), Paulo Alberto Lovatto(1), Cheila Roberta Lehnen(1), Amanda d'Ávila Carvalho(1), \\ Gerson Guarez Garcia(1) e Carlos Augusto Mallmann ${ }^{(2)}$
}

\begin{abstract}
(1)Universidade Federal de Santa Maria (UFSM), Dep. de Zootecnia, CEP 97105-900 Santa Maria, RS. E-mail: lucianoh@mail.ufsm.br, lovatto@smail.ufsm.br, cheilalehnen@yahoo.com.br, amanda@mail.ufsm.br, ggg@smail.ufsm.br (2)UFSM, Laboratório de Análises Micotoxicológicas. E-mail: mallmann@lamic.ufsm.br
\end{abstract}

\begin{abstract}
Resumo - Um experimento foi realizado para avaliar a digestibilidade de dietas e balanços metabólicos de suínos alimentados com dietas contendo zearalenona (ZEA) com e sem adição de organoaluminossilicato (OA). Foram utilizadas 12 leitoas com peso inicial de $12 \mathrm{~kg}$, alojadas em gaiolas metabólicas. O delineamento foi inteiramente casualizado, com três tratamentos (controle, controle +2 ppm de ZEA e controle +2 ppm de ZEA com adição de $0,3 \%$ de OA na dieta) e quatro repetições, com o animal como unidade experimental. A ZEA e o OA não influenciaram ( $\mathrm{p}>0,05)$ o consumo de matéria seca, a digestibilidade da matéria seca e energia bruta, metabolização da energia, proteína digestível e energias digestível e metabolizável das dietas. A ZEA e OA não alteraram o balanço do $N(p>0,05)$, mas modificaram $(p<0,05)$ a excreção fecal de $P$. Nas dietas contendo ZEA e ZEA+OA, a excreção fecal de $\mathrm{P}$ foi 15 e $10 \%$ menor do que no grupo controle. A ZEA e o OA não alteraram $(p>0,05)$ a absorção de $P$ em função da ingestão. O consumo de 2 ppm de ZEA com ou sem adição de $0,3 \%$ de OA não interfere na digestibilidade das dietas e no metabolismo dos suínos.
\end{abstract}

Termos para indexação: adsorvente, energia, micotoxinas, nitrogênio.

\section{Digestibility and metabolism of piglet diets containing zearalenone with addition of organoaluminosilicate}

\begin{abstract}
An experiment was carried out to asses diet digestibility and metabolism balance of piglets fed on diets containing zearalenone (ZEA) with and without addition of organaluminisilicate (OA). Twelve gilts littermates were used, with $12 \mathrm{~kg}$ initial body weight, housed in metabolic cages. A completely randomized design was used and comprised three treatments (control, control + 2 ppm ZEA, and control + 2 ppm ZEA with addition of $0.3 \%$ of OA), and four replications, being the animal as experimental unit. The ZEA and the OA did not affect ( $p>0.05)$ dry matter intake, digestibility of dry matter and gross energy, metabolizable energy, digestible energy as well as digestible protein. The nitrogen balance was not modified ( $p>0.05$ ) by ZEA and OA. However, it influenced $(\mathrm{p}<0.05)$ the fecal excretion of $\mathrm{P}$. In diets containing ZEA and ZEA+OA, fecal excretion of $\mathrm{P}$ was 15 and $10 \%$ lower than the control group. The ZEA and the OA did not affect $(\mathrm{p}>0.05)$ the absorption of $\mathrm{P}$ in relation to the ingestion. The intake of $2 \mathrm{ppm}$ of ZEA with or without addition of $0.3 \%$ of OA in diet does not affect the digestibility of diets and the metabolism of pigs.
\end{abstract}

Index terms: adsorbent, energy, mycotoxins, nitrogen.

\section{Introdução}

O Brasil é o terceiro maior produtor mundial de milho, com 40 milhões de toneladas de produção anual, das quais $28 \%$ são utilizadas na suinocultura (Sindirações, 2005). O cultivo em climas tropical e subtropical assim como a qualidade nutricional expõem o milho a problemas de contaminação por micotoxinas.

A zearalenona (ZEA) é uma das principais micotoxinas encontradas no milho, com regulamenta- ções específicas para as concentrações em cereais de 0,03 a 1 ppm em países como Áustria, Brasil, França, Itália, Rússia e Uruguai (FAO, 1995). A ZEA é um componente não esteroidal e um fitoestrógeno, que é um metabólito secundário de fungos do gênero Fusariun (Diekman \& Green, 1992). No milho produzido, principalmente, no centro-oeste do Brasil, esse fungo é mais freqüente que o Aspergillus flavus, responsável pela produção das aflatoxinas (Rodríguez-Amaya \& Sabino, 2002). O desenvolvimento do Fusarium e a produção 
de ZEA estão relacionados a temperaturas e umidade elevadas. O Fusarium modifica os componentes da parede celular e secreta enzimas de características digestivas, que alteram a composição nutricional dos grãos (Matthäus et al., 2004).

Os metabólitos da ZEA possuem atividades estrogênica e anabólica em suínos (Etienne \& Dourmad, 1994). Para exercer essas atividades, esses metabólitos utilizam receptores celulares que competem com o $17 \beta$-estradiol na ligação com receptores estrogênicos dos órgãos reprodutivos (Kuiper-Goodman et al., 1987). Esses aumentam a síntese de proteína, conseqüentemente, a secreção das células endometriais, a síntese das proteínas uterinas e o peso do trato reprodutivo se elevam (Dänicke et al., 2005).

Suínos são os animais mais sensíveis aos efeitos tóxicos da ZEA e, pode ocorrer hiperestrogenismo em doses acima de $1 \mathrm{ppm}$ de ZEA na dieta (Bennett \& Klich, 2003). A toxicidade da ZEA altera o sistema imunológico e interfere nos aspectos bioquímicos da lipogênese e lipólise (Diekman \& Green, 1992; Maaroufi et al., 1996).

Pela importância das micotoxicoses no desenvolvimento dos animais, várias alternativas têm sido estudadas a fim de minimizar intoxicações (Ramos \& Hernández, 1997). A mais utilizada é a física, que usa adsorventes não-nutritivos que se ligam às micotoxinas no trato gastrintestinal (Huwig et al., 2001). As argilas do tipo aluminossilicato, utilizadas como adsorventes de micotoxinas para suínos, apresentam elevada afinidade pelas aflatoxinas, mas reduzida afinidade pelas toxinas de menor polaridade como a ZEA (Avantaggiato et al., 2005). A incorporação de compostos orgânicos à superfície dos aluminossilicatos (organoaluminossilicato) modifica a composição química, o que aumenta a capacidade de adsorção da ZEA(Lemke et al., 1998). A eficiência dos organoaluminossilicatos na adsorção da ZEA tem sido avaliada somente in vitro, não considerando suas propriedades inespecíficas a esta toxina, como alterações digestivas e metabólicas em suínos. Vários estudos têm demonstrado as consequiências da ação estrogênica da ZEA no sistema reprodutivo de suínos (Diekman \& Green, 1992; Lawlor \& Lynch, 2001). Embora afete principalmente o sistema reprodutivo, o hiperestrogenismo causado pelaZEA também altera o metabolismo protéico, energético e mineral dos animais (Szkudelska et al., 2002; Abid-Essefi et al., 2004).

Os estudos in vivo são mais eficientes na avaliação da adsorção de micotoxinas e permitem avaliar a influência do adsorvente na digestibilidade de dietas e absorção de nutrientes (Avantaggiato et al., 2003). No entanto, os principais resultados foram obtidos com animais de laboratório.

O objetivo deste trabalho foi avaliar a digestibilidade das dietas e balanços metabólicos de suínos alimentados com dietas contendo zearalenona com ou sem adição de um organoaluminossilicato.

\section{Material e Métodos}

O experimento foi realizado de abril a maio de 2005 . Foram utilizados 12 fêmeas suínas, geneticamente homogêneas e meias-irmãs paternas, oriundas de cruzamentos industriais, com peso vivo médio inicial de $12 \mathrm{~kg}$. Os animais foram alojados em gaiolas metabólicas, mantidas em ambiente semi-controlado em temperatura média de $22^{\circ} \mathrm{C}$. O delineamento experimental foi o inteiramente casualizado com três tratamentos - dieta controle, controle $+2 \mathrm{ppm}$ de ZEA e controle $+2 \mathrm{ppm}$ de ZEA com adição de $0,3 \%$ de OA na dieta. Cada tratamento teve quatro repetições, e o animal foi a unidade experimental.

A ZEA foi produzida no Laboratório de Análises Micotoxicológicas (Lamic) da UFSM. A produção de ZEA foi realizada conforme o método de Jiménez et al. (1996). Em frascos erlenmeyer, adicionou-se água até que as amostras de milho obtivessem 0,97 de atividade de água. Os esporos do fungo foram produzidos em ágar batata cultivados a $25^{\circ} \mathrm{C}$ por cinco dias. Após esse período, os esporos foram lavados com água estéril e solubilizados à concentração de $1 \times 10^{0,6}$, sendo $1 \mathrm{~mL}$ aplicado no material de cultura. A incubação foi por quatro semanas, duas em temperatura de $28^{\circ} \mathrm{C}$ e duas a $15^{\circ} \mathrm{C}$. Em seguida, o material foi secado, triturado, quantificado e estocado a $-4^{\circ} \mathrm{C}$. A quantificação da ZEA foi realizada por cromatografia líquida de alta eficiência, com limite de quantificação de $12 \mu \mathrm{g} \mathrm{kg} \mathrm{kg}^{-1}$ e coeficiente de recuperação de $89 \%$. O coeficiente de correlação da curva de calibração variou de 0,995 a 0,999.

As dietas experimentais (Tabela 1) foram formuladas com utilização do modelo e das recomendações nutricionais do National Research Council (1998). O experimento durou 21 dias -5 dias de adaptação dos animais às gaiolas e ao alimento e 16 dias de coleta. As rações foram fornecidas de acordo com o peso metabólico $\left(\mathrm{PV}^{0,60}\right)$. A quantidade diária foi ajustada à estimativa do ganho médio diário e, considerou-se um consumo de 2,6 vezes a mantença estimada em $250 \mathrm{kcal} \mathrm{EM} / \mathrm{kg} \mathrm{PV}^{0,60}$ (Noblet et al., 1993). O alimento 
foi distribuído em três refeições diárias, às 8,13 e 18h, com os animais tendo livre acesso à água.

Foi utilizado o método de coleta total de fezes, sendo o início e o final das coletas determinados pelo aparecimento de fezes marcadas (foram adicionados $1,5 \%$ de $\mathrm{Fe}_{2} \mathrm{O}_{3}$ às dietas). As fezes totais foram coletadas duas vezes ao dia, acondicionadas em sacos de plástico e conservadas em congelador a $-10^{\circ} \mathrm{C}$. No final do experimento, as fezes foram homogeneizadas e amostradas $(0,5 \mathrm{~kg})$, secadas em estufa de ventilação forçada $\left(60^{\circ} \mathrm{C}\right.$ por 72 horas) e moídas para análises posteriores. A urina excretada era drenada para baldes de plástico com $25 \mathrm{~mL}$ de $\mathrm{HCl} 6 \mathrm{~N}$. A cada 12 horas, após homogeneização, o volume era medido e uma amostra de $5 \%$ era retirada e conservada sob refrigeração $\left(4^{\circ} \mathrm{C}\right)$. As análises químicas das fezes e da urina foram realizadas pelo método da Association of Official Analytical Chemists (1990). Foram avaliados o consumo de matéria seca (CMS), coeficiente de digestibilidade aparente da matéria seca $\left(\mathrm{CD}_{\mathrm{a}} \mathrm{MS}\right)$, coeficiente de digestibilidade aparente da energia bruta $\left(C D_{a} E B\right)$, coeficiente de metabolização da energia (CME), proteína digestível aparente $\left(\mathrm{PD}_{\mathrm{a}}\right)$, energias digestível $\left(\mathrm{ED}_{\mathrm{a}}\right)$ e metabolizável $\left(\mathrm{EM}_{\mathrm{a}}\right)$ aparentes, balanço do $\mathrm{N}$ e a digestibilidade do $\mathrm{P}$. Os valores de $\mathrm{CD}_{\mathrm{a}} \mathrm{MS}, \mathrm{CD}_{\mathrm{a}} \mathrm{EB}, \mathrm{CME}, \mathrm{PD}_{\mathrm{a}}, \mathrm{ED}_{\mathrm{a}}$ e $\mathrm{EM}_{\mathrm{a}}$ foram determinados de acordo com o método de Matterson et al. (1965).

Os dados foram submetidos à análise de variância, com efeitos da ZEA e do OA incluídos no modelo.

Tabela 1. Composição calculada da ração basal ${ }^{(1)}$.

\begin{tabular}{|c|c|}
\hline Ingredientes & Composição (\%) \\
\hline Milho & 59,82 \\
\hline Farelo de soja & 31,52 \\
\hline Óleo vegetal & 2,44 \\
\hline Premix $^{(2)}$ & 6,00 \\
\hline L-Lisina & 0,15 \\
\hline Metionina & 0,07 \\
\hline \multicolumn{2}{|l|}{ Valores calculados } \\
\hline Energia metabolizável $\left(\mathrm{kcal} \mathrm{kg}^{-1}\right)$ & 3.265 \\
\hline Proteína bruta (\%) & 20,90 \\
\hline Cálcio (\%) & 0,83 \\
\hline Fósforo total (\%) & 0,59 \\
\hline Lisina $(\%)$ & 1,15 \\
\hline Metionina (\%) & 0,30 \\
\hline Treonina (\%) & 0,74 \\
\hline \multicolumn{2}{|c|}{$\begin{array}{l}{ }^{(1)} \text { Matéria natural. }{ }^{(2)} \text { Composição por } \mathrm{kg}: 130 \mathrm{~g} \text { de Ca, } 55 \mathrm{~g} \text { de P, } 36 \mathrm{~g} \text { de } \\
\mathrm{Na}, 21 \mathrm{~g} \text { de metionina, } 45 \mathrm{~g} \text { de lisina, } 160.000 \text { UI Vit. A, } 30.000 \text { UI Vit. } \\
\mathrm{D} 3,1.000 \mathrm{mg} \text { Vit. E, } 40 \mathrm{mg} \text { Vit. K3, } 32 \mathrm{mg} \text { Vit. B1, } 100 \mathrm{mg} \text { Vit. B2, } 40 \\
\mathrm{mg} \text { de Vit. B6, } 400 \mathrm{mg} \text { de Vit. B12, } 16 \mathrm{mg} \text { de ácido fólico, } 260 \mathrm{mg} \text { de } \\
\text { ácido pantotênico, } 2 \mathrm{mg} \text { de biotina, } 5.000 \mathrm{mg} \text { de colina, } 520 \mathrm{mg} \mathrm{de} \\
\text { niacina, } 180 \mathrm{mg} \mathrm{de} \mathrm{Cu,} 1.320 \mathrm{mg} \text { de Fe, } 8 \mathrm{mg} \text { de I, } 300 \mathrm{mg} \mathrm{de} \mathrm{Mg,} 6 \mathrm{mg} \\
\text { de Se, } 1.920 \mathrm{mg} \text { de Zn, } 1 \mathrm{~g} \text { de promotor de crescimento, } 549 \mathrm{mg} \mathrm{de} \\
\text { antioxidante e } 0,5 \mathrm{~g} \text { de } \mathrm{F} \text {. }\end{array}$} \\
\hline
\end{tabular}

As análises estatísticas foram realizadas por meio do SAS Institute (2000).

\section{Resultados e Discussão}

Os teores nutricionais das dietas não foram alterados pela presença de ZEA (Tabela 2). O fungo Fusarium, quando presente em cereais, secreta amilases, celulases, proteases e xilanases, enzimas que modificam os componentes da parede celular e alteram o perfil nutricional do grão (Matthäus et al., 2004). Entretanto, não existem relatos sobre o fato de que a contaminação de dietas com ZEA purificada, sem presença do fungo, altere a composição nutricional dos ingredientes.

A presença de ZEA, com ou sem adição de OA, não alterou $(p>0,05)$ o consumo de matéria seca, a digestibilidade da matéria seca e energia bruta, metabolização da energia, e energias digestível e metabolizável aparente das dietas (Tabela 3).

A camada epitelial do intestino é a primeira barreira que previne a entrada de agentes patógenos e a sua integridade é mantida por estruturas intercelulares organizadas (Bouhet \& Oswald, 2005). Alguns fatores

Tabela 2. Composição analisada das rações experimentais.

\begin{tabular}{lccc}
\hline Nutriente & \multicolumn{3}{c}{ Tratamentos } \\
\cline { 2 - 4 } & Controle & ZEA $^{(1)}$ & ZEA + OA \\
\hline Matéria seca (\%) & 87,17 & 87,08 & 87,27 \\
Proteína bruta (\%) & 22,41 & 22,47 & 22,35 \\
Extrato etéreo (\%) & 9,65 & 8,51 & 9,57 \\
Fósforo total (\%) & 0,80 & 0,69 & 0,69 \\
Energia bruta (kcal kg-1) & 4.960 & 4.832 & 4.855 \\
\hline
\end{tabular}

${ }^{(1)}$ Zearalenona. ${ }^{(2)}$ Zearalenona + organoaluminossilicato.

Tabela 3. Consumo de matéria seca, coeficientes de digestibilidade aparente da matéria seca e energia, metabolização da energia, proteína digestível aparente, energias digestível e metabolizável aparentes de dietas para suínos, com 2 ppm de zearalenona (ZEA), com ou sem adição de $0,3 \%$ de organaluminossilicato (OA).

\begin{tabular}{|c|c|c|c|c|c|}
\hline \multirow[t]{2}{*}{ Variáveis } & \multicolumn{3}{|c|}{ Tratamentos } & \multirow{2}{*}{ epr ${ }^{(1)}$} & \multirow[t]{2}{*}{$\mathrm{P}^{(2)}$} \\
\hline & Controle & ZEA & $\mathrm{ZEA}+\mathrm{OA}$ & & \\
\hline \multicolumn{6}{|l|}{ Consumo } \\
\hline Matéria seca $\left(\mathrm{kg} \mathrm{dia}^{-1}\right)$ & 0,76 & 0,74 & 0,76 & 0,01 & 0,17 \\
\hline \multicolumn{6}{|l|}{ Coeficiente de digestibilidade } \\
\hline Matéria seca (\%) & 80,65 & 81,54 & 84,42 & 1,02 & 0,06 \\
\hline Energia bruta (\%) & 81,81 & 81,82 & 84,92 & 0,96 & 0,07 \\
\hline \multicolumn{6}{|l|}{ Coeficiente de metabolização } \\
\hline Energia bruta (\%) & 81,81 & 81,82 & 84,91 & 0,96 & 0,07 \\
\hline \multicolumn{6}{|l|}{ Proteína e energia da dieta } \\
\hline Proteína digestível (\%) & 17,10 & 17,09 & 17,97 & 0,28 & 0,09 \\
\hline Energia digestível $\left(\mathrm{kcal} \mathrm{kg}^{-1}\right)$ & 4.058 & 3.954 & 4.057 & 47,39 & 0,09 \\
\hline Energia metabolizável $\left(\mathrm{kcal} \mathrm{kg}^{-1}\right)$ & 3.992 & 3.903 & 4.057 & 46,67 & 0,12 \\
\hline
\end{tabular}


químicos como hormônios, neurotransmissores, proteases e micotoxinas podem modificar essa estrutura, o que altera a diferenciação das células do epitélio intestinal (Bouhet \& Oswald, 2005). Isso afeta a síntese enzimática e a morfologia epitelial, que altera a digestibilidade e a absorção de nutrientes. Embora a ZEA não interfira neste processo de diferenciação epitelial, a contaminação natural ocorre em conjunto com o deoxinivalenol, que altera o epitélio intestinal e a digestibilidade das dietas (Dänicke et al., 2004a; Bouhet \& Oswald, 2005). O uso unicamente de ZEA neste experimento, sem deoxinivalenol, provavelmente não alterou o epitélio intestinal dos animais.

Os metabólitos da zearalenona ( $\alpha$ e $\beta$ zeranol) são semelhantes ao estradiol e seus mecanismos de ação incluem a redução da lipogênese via restrição do metabolismo da glicose para acetil-CoA (Szkudelska et al., 2002). A oxidação de lipídios também é reduzida pelos metabólitos da ZEA em doses acima de $1 \mathrm{ppm}$ (Kandulska et al., 1999). Essa redução pode induzir à oxidação de outros substratos energéticos como rota metabólica compensatória e alterar o metabolismo energético. Por isso, com a redução de reservas energéticas, principalmente glicogênica, há aumento do consumo de matéria seca para regular o metabolismo energético (Friedman et al., 1999).

Em razão das semelhanças fisiológicas, estudos realizados com roedores podem ser transpostos aos suínos, e podem elucidar aspectos digestivos e metabólicos (Jørgensen et al., 1997). A dose de 2 ppm de ZEA utilizada neste experimento é considerada elevada para roedores. No entanto, em suínos, não altera o consumo de matéria seca e a eficiência na metabolização da energia. Estes resultados podem estar relacionados ao estado fisiológico em que se encontravam os animais, pois, os suínos em fase de crescimento apresentam baixa deposição de lipídios antes de atingirem o potencial máximo de deposição protéica (Van Milgen \& Noblet, 1999). Efeitos negativos da ZEA no metabolismo lipídico, e conseqüentemente energético, podem ter influência somente em suínos com maior deposição lipídica.

Os OA, por formarem complexos com os ácidos biliares no intestino delgado quando em doses acima de $0,4 \%$ na dieta, interferem na digestibilidade da gordura e, conseqüentemente, da energia (Döll et al., 2005). Em dietas que contêm adsorventes com atividade enzimática intrínseca, a digestibilidade da gordura também é reduzida (Dänicke et al., 2004b). No entanto, a adição de $0,3 \%$ de $\mathrm{OA}$ na dieta não alterou a digestibilidade da energia, o que não modificou a energia digestível e metabolizável das dietas. Os efeitos dos adsorventes na digestibilidade e metabolizibilidade, portanto, estão relacionados à sua composição e concentração na dieta.

Não houve influência da ZEA e do OA $(\mathrm{p}>0,05)$ sobre as estimativas de excreções fecal e urinária, absorção, retenção e retenção em função da absorção de $\mathrm{N}$ (Tabela 4). A presença de ZEA na dieta não alterou a retenção de $\mathrm{N}$ dos animais em razão de os metabólitos da ZEA fixarem-se somente a receptores do trato reprodutivo, o que não altera a deposição protéica dos demais órgãos. A deposição protéica apresenta correlação positiva com a retenção de N (Hennig et al., 1982) e é considerada um dos principais componentes químicos no ganho de peso de suínos (De Lange et al., 2003). Os resultados do metabolismo do N, deste trabalho, portanto, explicam, em parte, os estudos conduzidos com leitoas, em que não se observou influência da ZEA no desempenho (Green et al., 1990).

A adição de OA nas dietas aumenta a concentração de albumina e as atividades de algumas enzimas relacionadas à síntese protéica em suínos (Döll et al., 2005). Por estarem relacionadas a esta síntese, as alterações destes componentes afetam o balanço do $\mathrm{N}$ (Hennig et al., 1982). No entanto, o balanço do $\mathrm{N}$ não foi influenciado pela adição de $\mathrm{OA}$ em dietas contaminadas com ZEA, neste trabalho.

A ingestão de $\mathrm{P}$ foi maior $(\mathrm{p}<0,05)$ no grupo controle e, no ajuste das demais variáveis, utilizou-se esta estimativa como co-variável (Tabela 5). A presença de ZEA e a adição de OA influenciaram $(\mathrm{p}<0,05)$ a excreção fecal de $P$.

Tabela 4. Balanço do nitrogênio de leitoas alimentadas com dietas contendo 2 ppm de zearalenona (ZEA), com ou sem adição de $0,3 \%$ de organoaluminossilicato (OA).

\begin{tabular}{|c|c|c|c|c|c|}
\hline \multirow[t]{2}{*}{ Variáveis } & \multicolumn{3}{|c|}{ Tratamentos } & \multirow[t]{2}{*}{$\mathrm{epr}^{(1)}$} & \multirow[t]{2}{*}{$\mathrm{p}^{(2)}$} \\
\hline & Controle & ZEA & $\mathrm{EA}+\mathrm{OA}$ & & \\
\hline \multicolumn{6}{|l|}{ Nitrogênio } \\
\hline Ingerido $\left(\mathrm{g} \mathrm{dia}^{-1}\right)$ & 27,56 & 26,61 & 27,76 & 0,46 & 0,23 \\
\hline Fezes $\left(\mathrm{g} \mathrm{dia}^{-1}\right)$ & 6,51 & 6,36 & 5,43 & 0,31 & 0,10 \\
\hline Urinário $\left(\mathrm{g} \mathrm{dia}^{-1}\right)$ & 7,18 & 5,47 & 7,10 & 0,11 & 0,07 \\
\hline Absorvido $^{(3)}\left(\mathrm{g} \mathrm{dia}^{-1}\right)$ & 21,05 & 20,25 & 22,33 & 0,59 & 0,12 \\
\hline Retido $\left(\mathrm{g} \mathrm{dia}^{-1}\right)$ & 13,87 & 14,78 & 15,23 & 0,68 & 0,09 \\
\hline Retido/absorvido (\%) & 65,89 & 79,98 & 68,20 & 2,58 & 0,10 \\
\hline
\end{tabular}

${ }^{(1)}$ Erro-padrão residual. ${ }^{(2)}$ Nível de significância. ${ }^{(3)}$ Nitrogênio ingerido utilizado como co-variável. 
Tabela 5. Fósforo ingerido, excretado nas fezes, absorvido, e absorvido em função do ingerido, de leitoas alimentadas com dietas contendo 2 ppm de zearalenona, com ou sem adição de $0,3 \%$ de organoaluminossilicato $(\mathrm{OA})^{(1)}$.

\begin{tabular}{lrrrrr}
\hline Variáveis & \multicolumn{3}{c}{ Tratamentos } & $\operatorname{epr}^{(2)}$ & $\mathrm{P}^{(3)}$ \\
\cline { 2 - 4 } & Controle & ZEA & ZEA + OA & & \\
\hline Fósforo & $6,17 \mathrm{a}$ & $5,09 \mathrm{~b}$ & $5,38 \mathrm{~b}$ & 0,10 & 0,00 \\
$\quad$ Ingerido $\left(\mathrm{g} \mathrm{dia}^{-1}\right)$ & $1,38 \mathrm{a}$ & $1,17 \mathrm{~b}$ & $1,24 \mathrm{a}$ & 0,12 & 0,05 \\
Fezes $^{(4)}\left(\mathrm{g} \mathrm{dia}^{-1}\right)$ & 4,79 & 3,92 & 4,14 & 0,12 & 0,06 \\
Absorvido $^{(4)}\left(\mathrm{g} \mathrm{dia}^{-1}\right)$ & 77,63 & 77,01 & 76,95 & 2,67 & 0,06 \\
\hline
\end{tabular}

(1) Médias com letras distintas na mesma linha diferem entre si pelo teste de Tukey $(\mathrm{p}<0,05)$. ${ }^{(2)}$ Erro-padrão residual. ${ }^{(3)}$ Nível de significância ${ }^{(4)}$ Fósforo ingerido utilizado como co-variável.

Os animais alimentados com a dieta contendo ZEA e com ZEA+OA excretaram 15 e $10 \%$ menos $\mathrm{P}$ nas fezes em relação ao grupo controle. No $\mathrm{P}$ absorvido em função do ingerido, não houve diferença $(p>0,05)$ entre os tratamentos. Por ser um dos fitoestrógenos de maior afinidade com o $17 \beta$-estradiol, a ZEA pode atuar na homeostasia dos minerais, com diminuição da excreção e aumento da retenção (Draper et al., 1997). O mecanismo completo da ação de agentes estrogênicos no metabolismo mineral ainda é desconhecido, principalmente em relação à digestão e absorção de minerais em suínos. Embora não se encontre registros sobre a influência dos OA no metabolismo do $\mathrm{P}$, os dados deste experimento demonstram tendência de alterações na absorção em função da ingestão de $\mathrm{P}$, com valores menores para a dieta com ZEA+OA.

As consequiências reprodutivas da exposição de suínos à ZEA são bem conhecidas, contudo pouca atenção tem sido dada aos parâmetros não reprodutivos. Apesar de estar consolidado o efeito da ZEA no trato gastrointestinal e no metabolismo de animais de laboratório, os dados deste trabalho representam uma contribuição aos estudos sobre o seu efeito na digestibilidade e no metabolismo de suínos. Embora os OA sejam excelentes adsorventes de micotoxinas, a atuação deles na digestibilidade e na absorção de nutrientes, especificamente, precisa ser complementada por estudos in vivo.

\section{Conclusões}

1. Os coeficientes de digestibilidade da matéria seca, proteína e energia bruta, metabolização da energia bruta, energias digestível e metabolizável aparente não são alterados em dietas com 2 ppm de zearalenona, com ou sem adição de $0,3 \%$ de organoaluminossilicato na dieta.

2. O balanço do nitrogênio de leitoas não é influenciado pela ingestão de $2 \mathrm{ppm}$ de zearalenona, com ou sem adição de $0,3 \%$ de organoaluminossilicato na dieta.

3. A digestibilidade do fósforo não é alterada pela presença de $2 \mathrm{ppm}$ de zearalenona, com ou sem adição de $0,3 \%$ de organoaluminossilicato na dieta.

4. A alimentação de leitoas com $2 \mathrm{ppm}$ de zearalenona diminui a excreção fecal de fósforo.

\section{Agradecimentos}

Ao CNPq e à Capes, pela concessão de bolsa; ao Laboratório de Análises Micotoxicológicas, pelo fornecimento das micotoxinas e realização das análises.

\section{Referências}

ABID-ESSEFI, S.; OUANES, Z.; HASSEN, W.; BAUDRIMONT, I.; CREPPY, E.; BACHA, H. Cytotoxicity, inhibition of DNA and protein syntheses and oxidative damage in cultured cells exposed to zearalenone. Toxicology in Vitro, v.18, p.467-474, 2004.

ASSOCIATION OF OFFICIAL ANALYTICAL CHEMISTS (Arlington, Estados Unidos). Official methods of analysis of the Association of Official Analytical Chemists. $15^{\text {th }}$ ed. Arlington, 1990. 1117 p.

AVANTAGGIATO, G.; HAVENAAR, R.; VISCONTI, A. Assessing the zearalenone-binding activity of adsorbent materials during passage through a dynamic in vitro gastrointestinal model. Food and Chemical Toxicology, v.41, p.1283-1290, 2003.

AVANTAGGIATO, G.; SOLFRIZZO, M.; VISCONTI, A. Recent advances on the use of adsorbent materials for detoxification of Fusarium mycotoxins. Food Additives and Contaminants, v.22, p.379-388, 2005.

BENNETT, J.W.; KLICH, M. Mycotoxins 10.1128/CMR.16.3.497516.2003. Clinical Microbiology Reviews, v.16, p.497-516, 2003. BOUHET, S.; OSWALD, I.P. The effects of mycotoxins, fungal food contaminants, on the intestinal epithelial cell-derived innate immune response. Veterinary Immunology and Immunopathology, v.108, p.199-209, 2005.

DÄNICKE, S.; SWIECH, E.; BURACZEWSKA, L.; UEBERSCHAR, K.H. Kinetics and metabolism of zearalenone in young female pigs. Journal of Animal Physiology and Animal Nutrition, v.89, p.268-276, 2005.

DÄNICKE, S.; VALENTA, H.; DÖLL, S.; GANTER, M.; FLACHOWSKY, G. On the effectiveness of a detoxifying agent in preventing fusario-toxicosis in fattening pigs. Animal Feed Science and Technology, v.114, p.141-157, 2004b.

DÄNICKE, S.; VALENTA, H.; KLOBASA, F.; DÖLL, S.; GANTER, M.; FLACHOWSKY, G. Effects of graded levels of 
Fusarium toxin contaminated wheat in diets for fattening pigs on growth performance, nutrient digestibility, deoxynivalenol balance and clinical serum characteristics. Archives of Animal Nutrition, v.58, p.1-17, 2004a.

DE LANGE, C.F.M.; MOREL, P.C.H.; BIRKETT, S.H. Modeling chemical and physical body composition of the growing pig. Journal of Animal Science, v.81, p.E159-E165, 2003.

DIEKMAN, M.A.; GREEN, M.L. Mycotoxins and reproduction in domestic livestock. Journal of Animal Science, v.70, p.1615$1627,1992$.

DÖLL, S.; GERICKE, S.; DÄNICKE, J.; RAILA, K.-H.; UEBERSCHÄR, R.; VALENTA, H.; SCHNURRBUSCH, U.; SCHWEIGERT, F.G.; FLACHOWSKY, G. The efficacy of a modified aluminosilicate as a detoxifying agent in Fusarium toxin contaminated maize containing diets for piglets. Journal of Animal Physiology and Animal Nutrition, v.89, p.342-358, 2005.

DRAPER, C.R.; EDEL, M.J.; DICK, I.M.; RANDALL, A.G.; MARTIN, G.B.; PRINCE, R.L. Phytoestrogens reduce bone loss and bone resorption in ophorectomized rats. Journal of Nutrition, v.127, p.1795-1799, 1997.

ETIENNE, M.; DOURMAD, J.-Y. Effects of zearalenone or glucosinolates in the diet on reproduction in sows: a review. Livestock Production Science, v.40, p.99-113, 1994.

FAO. Worldwide regulations for mycotoxins. Rome, 1995. 43p. (FAO. Food and nutrition paper, 64).

FRIEDMAN, M.I.; HARRIS, R.B.; JI, H.; RAMIREZ, I.; TORDOFF, M.G. Fatty acid oxidation affects food intake by altering hepatic energy status. American Journal of Physiology: Regulatory, Integrative and Comparative Physiology, v.276, p.10461053, 1999.

GREEN, M.L.; DIEKMAN, M.A.; MALAYER, J.R.; SCHEIDT, A.B.; LONG, G.G. Effect of prepubertal consumption of zearalenone on puberty and subsequent reproduction of gilts. Journal of Animal Science, v.68, p.171-178, 1990.

HENNIG, U.; WUNSCHE, J.; MEINL, M.; BORGMANN, E.; KREIENBRING, F; BOCK, H.D. Effect of graded protein supply at high-energy level on the fattening performance and the retention and utilization of feed energy, protein and amino acids by female fattening swine. 3. $\mathrm{N}$ retention and $\mathrm{N}$ and lysine metabolism determined by $\mathrm{N}$ balance and $\mathrm{N}$ analysis of the carcasses. Archive für Tierernährung, v.32, p.637-649, 1982.

HUWIG, A.; FREIMUND, S.; KAPPELI, O.; DUTLER, H. Mycotoxin detoxication of animal feed by different adsorbents. Toxicology Letters, v.122, p.179-188, 2001.

JIMÉNEZ, M.; MÁÑEZ, M.; HERNÁNDEZ, E.I. Influence of water activity and temperature on the production of zearalenone in corn by three Fusarium species. International Journal of Food Microbiology, v.29, p.417-421, 1996.

JØRGENSEN, H.; GABERT, V.M.; EGGUM, B.O. The nutritional value of high-lysine barley determined in rats, young pigs and growing pigs. Journal of the Science of Food and Agriculture, v.73, p.287295, 1997.
KANDULSKA, K.; NOGOWSKI, L.; SZKUDELSKI, T. Effect of some phytoestrogens on metabolism of rat adipocytes. Reproduction, Nutrition, Development, v.39, p.497-501, 1999.

KUIPER-GOODMAN, T.; SCOTT, P.M.; WATANABE, H. Risk assessment of the mycotoxin zearalenone. Regulatory Toxicology and Pharmacology, v.7, p.253-306, 1987.

LAWLOR, P.G.; LYNCH, P.B. Mycotoxins in pig feeds. 2. Clinical aspects. Irish Veterinary Journal, v.54, p.172-176, 2001.

LEMKE, S.; GRANT, P.G.; PHILLIPS, T.D. Adsorption of zearalenone by organophilic montmorillonite clay. Journal of Agricultural and Food Chemical, v.46, p.3789-3796, 1998.

MAAROUFI, K.; CHEKIR, L.; CREPPY, E.E.; ELLOUZ, F.; BACHA, H. Zearalenone induces modifications of hematological and biochemical parameters in rats. Toxicon, v.34, p.535-540, 1996.

MATTERSON, L.D.; POTTER, L.M.; STUTZ, M.W.; SINGSEN, E.P. The metabolizable energy of feed ingredients for chickens. Storrs: The University of Connecticut, Agricultural Experiment Station, 1965. 11p. (Research report, 7).

MATTHÄUS, K.; DÄNICKE, S.; VAHJEN, W.; SIMON, O.; WANG, J.; VALENTA, H.; MEYER, K.; STRUMPF, A.; ZIESENIß, H.; FLACHOWSKY, G. Progression of mycotoxin and nutrient concentrations in wheat after inoculation with Fusarium culmorum. Archives of Animal Nutrition, v.58, p.19-35, 2004.

NOBLET, J.; SHI, X.S.; DUBOIS, S. Metabolic utilization of dietary energy and nutrients for maintenance energy requirements in sows: basis for a net energy system. British Journal of Nutrition, v.70, p.407-419, 1993.

NATIONAL RESEARCH COUNCIL (Washington, DC). Nutrient requirements of swine. $10^{\text {th }}$ ed. Washington: National Academy of Science, 1998. 189p.

RAMOS, A.J.; HERNÁNDEZ, E. Prevention of aflatoxicosis in farm animals by means of hydrated sodium calcium aluminosilicate addition to feedstuffs: a review. Animal Feed Science and Technology, v.65, p.197-206, 1997.

RODRÍGUEZ-AMAYA, D.B.; SABINO, M. Pesquisa em micotoxinas no Brasil: a última década em foco. Brazilian Journal of Microbiology, v.33, p.1-11, 2002.

SAS INSTITUTE (Cary, Estados Unidos). SAS software: user's guide, version 8.2. Cary, 2000. 291p.

SINDIRAÇÕES. Demanda de macronutrientes. Disponível em: <http://www.sindiracoes.org.br>. Acesso em: 20 dez. 2005.

SZKUDELSKA, K.; SZKUDELSKI, T.; NOGOWSKI, L. Daidzein, coumestrol and zearalenone affect lipogenesis and lipolysis in rat adipocytes. Phytomedicine, v.9, p.338-345, 2002.

VAN MILGEN, J.; NOBLET, J. Energy partitioning in growing pigs: the use of a multivariate model as an alternative for the factorial analysis. Journal of Animal Science, v.77, p.2154-2162, 1999.

Recebido em 7 de abril de 2006 e aprovado em 26 de outubro de 2006 